\title{
Improving H.E.S.S. cosmic-ray background rejection by means of a new Gamma-Ray Air Shower Parametrisation (GRASP)
}

\author{
Andreas Hillert ${ }^{a}$, Francois Brun ${ }^{* b}$ and Robert Parsons ${ }^{a}$ \\ ${ }^{a}$ Max Planck Institute for Nuclear Physics, Heidelberg, Germany \\ ${ }^{b}$ Irfu, CEA-Saclay, France \\ E-mail: andreas.hillertempi-hd.mpg.de, francois.brunecea.fr, \\ daniel.parsons@mpi-hd.mpg.de
}

\begin{abstract}
Imaging Atmospheric Cherenkov Telescopes (IACTs), with their high sensitivity and large fieldof-views, are ideal instruments to study the universe in VHE $\gamma$-rays. IACTs image Cherenkov light emitted by $\gamma$-rays from induced particle cascades, developing in the atmosphere. The sensitivity of the IACTs depends critically on their capability to reduce the background caused by the much more numerous hadronic air showers, induced from the hadronic component of the cosmic rays. In this work we apply a new image analysis method using an advanced Gamma Ray Air Shower Parametrisation (GRASP) to data from the High Energy Stereoscopic System (H.E.S.S. Phase I). The new method provides additional classifying variables for a decision tree based learning algorithm to differentiate $\gamma$-ray induced air showers from hadronic ones. When combined with a (recently developed) $\gamma$-ray reconstruction method that uses an Image Pixel-wise fit for Atmospheric Cherenkov Telescopes (ImPACT), a significant improvement in sensitivity is observed compared to the H.E.S.S. Standard Analysis.
\end{abstract}

The 34th International Cosmic Ray Conference,

30 July- 6 August, 2015

The Hague, The Netherlands

${ }^{*}$ Speaker. 


\section{Introduction}

The very high-energy $\gamma$-ray astronomy is defined as astronomy in the regime for energies even greater than $100 \mathrm{GeV}$. In this regime the key critical point in the detection of sources and physical information obtainable is the ability to discriminate the $\gamma$-rays from the hadronic cosmic ray background as well as the accurate reconstruction of their physical properties. The higher the efficiency at which this background rejection works the faster one can detect a source at a predetermined significance level. The background discrimination technique typically used dates back to when the Whipple observatory began using the imaging technique and when it was shown by Hillas [1], that it should be indeed possible. This so called Hillas method of discrimination between $\gamma$-rays and hadronic background uses the width, length and orientation of the Cherenkov light image of the shower inside a camera and was very successful. However, this method suffers from a number of drawbacks. The non-linear mapping of Cherenkov light has the effect that the image gets expanded on one side whereas on the other side it gets compressed, yielding an asymmetric image. This leads to a significant uncertainty in the determination of the image axis and therefore the shower direction. Furthermore, the second moments depend on the cleaning method of the image, which introduce a systematic error. This paper will introduce a new parametrisation of the shower image which remedies all the drawbacks mentioned before and shows a significant improvement in sensitivity of the H.E.S.S. Phase I instrument.

\section{GRASP - Gamma Ray Air Shower Parametrisation}

This section will introduce an alternative reconstruction algorithm that applies a fit of pixel amplitudes to an expected image obtained from a Gamma Ray Air Shower Parametrisation (GRASP). This parametrisation was obtained using Monte Carlo air shower simulations, from the CORSIKA (COsmic Ray SImulations for KAscade) software [2], by parameterising the angular Cherenkov photon distribution with suitable analytical functions. In turn those functions depend on air shower parameters like the impact distance or the height of the first interaction in the atmosphere. For further details see [3].

\subsection{Shower image and profiles}

The simulation of a $1 \mathrm{TeV}$ gamma-ray air shower from CORSIKA is shown in Fig. 1. It shows the angular distribution of photons averaged over 50 shower events with an impact distance of $200 \mathrm{~m}$ and a height of the first interaction of $1 X_{0}$ (radiation length in air). From this illustration the basic properties of a better parametrisation can be inferred by looking at the longitudinal and transverse part of the shower image. In order to extract the longitudinal and transversal angular distribution of photons, slices in $u$ and $v$ are made. As an example the shower event displayed in Fig. 1 is taken and a slice along the $u$-axis is made. From the resulting distributions corresponding to the longitudinal and transverse slice of the image, suitable analytical functions need to be found in order to model the shower image. The blue, black and red line in Fig. 2 (left) correspond to a fit of a Moyal function of the form:

$$
L_{\text {Moyal }}(\lambda, n, l)=\frac{n}{l \cdot \sqrt{2 \pi}} \cdot \exp \left(-\frac{1}{2}(\lambda+\exp (-\lambda))\right)
$$




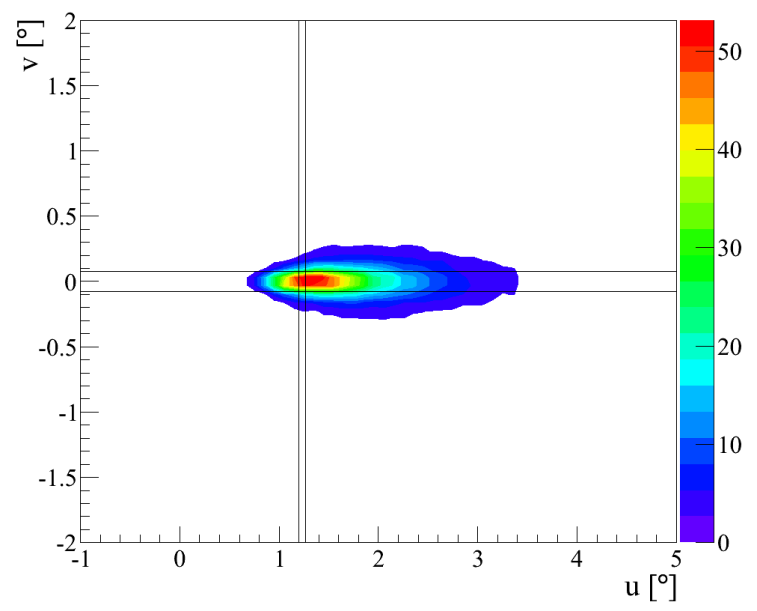

Figure 1: Shower image for an averaged shower over 50 shower events with an energy of $1 \mathrm{TeV}$, an impact distance of $200 \mathrm{~m}$ and a height of the first interaction of $1 X_{0}$ (radiation length in air). The black solid lines illustrate the slices that were made to find the analytical functions for the longitudinal and transverse part of the shower image.
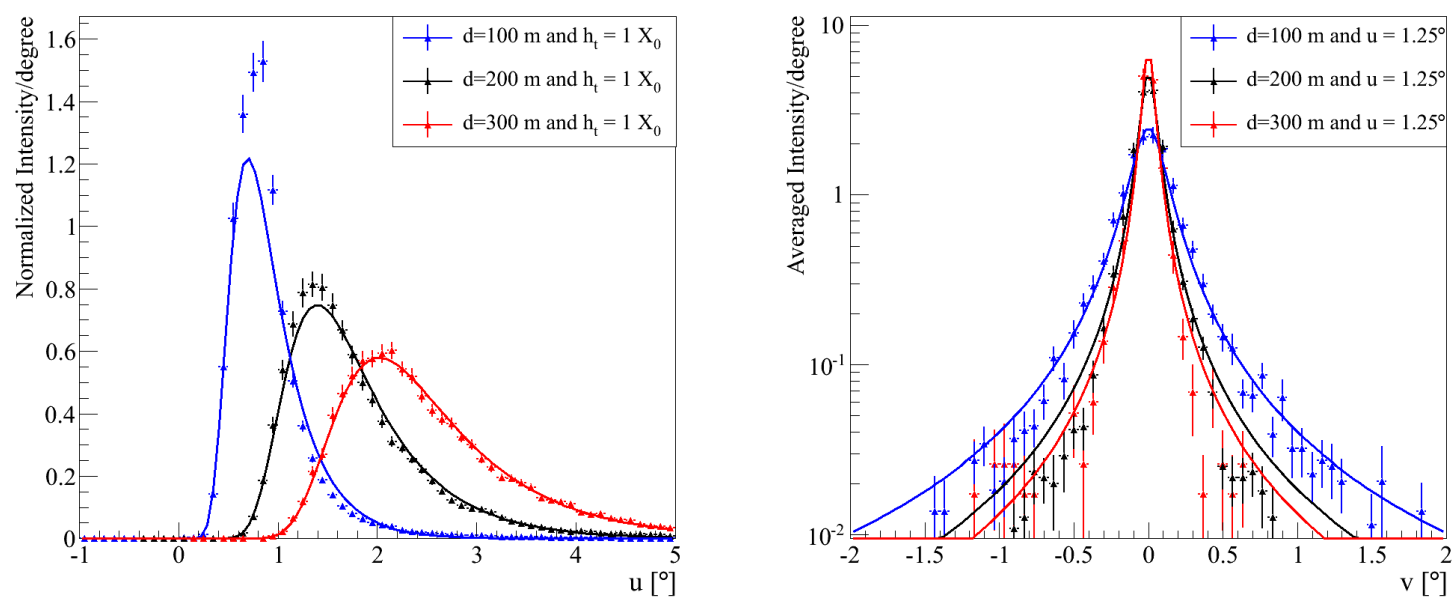

Figure 2: On the left the longitudinal distribution of photons converted to photoelectrons is shown for the same $h_{t}=1 X_{0}$ but different impact distances of $\mathrm{d}=100 \mathrm{~m}, \mathrm{t}=200 \mathrm{~m}$ and $\mathrm{d}=300 \mathrm{~m}$. On the right the transversal distribution is shown for the same three impact distances.

with $\lambda=\frac{u-p}{l}$. Here, $n$ describes the normalisation, $p$ the peak and $l$ the length of the Moyal function. It can be seen that this function describes the longitudinal profile quite well. The right side of Fig. 2 shows the transversal profile, which can be very well represented by a Lorentz function of the form:

$$
T_{\text {Lorentz }}=a \frac{w}{\pi\left(w^{2}+v^{2}\right)}
$$

where $a$ is the normalisation and $w$ is the width of the function. This clearly shows that a parametrisation that should describe the longitudinal distribution of the shower depends on the impact dis- 
tance as well as the height of the first interaction in the atmosphere. For the transverse part, by looking at Fig. 1, it is evident that the width of the shower depends on where the slice is made or in other words the longitudinal position $u$. This is due to the air shower broadening while developing in the atmosphere. Furthermore, the width also depends on the impact distance illustrated in Fig. 2. The peak of the longitudinal projection of Cherenkov photons corresponds to the maximum intensity from the height of the shower maximum $h_{\max }$ seen under the angle $\phi\left(h_{\max }\right)=\phi_{\max } \approx d / h_{\max }$, where $d$ is the impact distance of the event. By combining the equations (2.1) and (2.2) for the longitudinal and transverse part, the total Cherenkov image due to a $\gamma$-ray induced air shower can be described by the following formula:

$$
\begin{aligned}
\frac{\rho(u, v)}{\mathrm{d} u \mathrm{~d} v} & =L_{\text {Moyal }}(u ; p, l, n) \\
& \times \frac{k \cdot w(u, d)}{\pi\left(k \cdot w(u, d)^{2}+v^{2}\right)}\left[\text { p.e./degrees }{ }^{2}\right]
\end{aligned}
$$

It describes the angular distribution of Cherenkov photons inside the solid angle $\mathrm{d} u \mathrm{~d} v$. In order to get the total photoelectrons seen by one pixel of the camera this parametrisation has to be integrated over the pixel area. The shower image resulting from this parametrisation provides a number of photo electrons per degree squared and by integrating it over one pixel the expected intensity in photoelectrons can be calculated. The basic form of the probability density function to observe a signal given an expected intensity can be found in [4] as well as the log-likelihood function. The log-likelihood for each shower image is then minimized using the MINUIT tool [5] inside the ROOT software [6]. The fitting procedure yields the peak $p$ and length $l$ of the shower image as well as a scaling factor $k$ to the Lorentz width $w$, which defines the compatibility of the shower image being produced from gamma-rays. Furthermore, the normalisation for this fit was determined by calculating the ratio between the total observed image intensity to the total intensity expected from integrating the parametrisation over the entire camera. The Lorentz width distribution $w(u, d)$ as a function of longitudinal position and impact distance is obtained from CORSIKA simulations [3].

\subsection{Training and Evaluation of the BDT method}

The Hillas based $\gamma$-hadron separation is described in [1]. The basis for the background rejection method used in this work is the multivariate analysis technique developed in [7]. For the training simulated $\gamma$-rays were used resulting from a point source and follow an energy distribution of $\frac{d N}{d E} \propto E^{-\Gamma}$ with a spectral index $\Gamma=2.0$. The Off-Events consist of cosmic-rays from data which reach the earth isotropically and are therefore homogeneously distributed over the field of view of the camera. New parameters from the GRASP method were used and combined with some of the Hillas parameters in [7]. There are several parameters which yield a potential for $\gamma$-hadron separation:

- Moyal peak $p$

- Moyal length $l$

- Lorentz width scaling factor $k$

- Moyal length times Lorentz width scaling factor $l \times k$ 

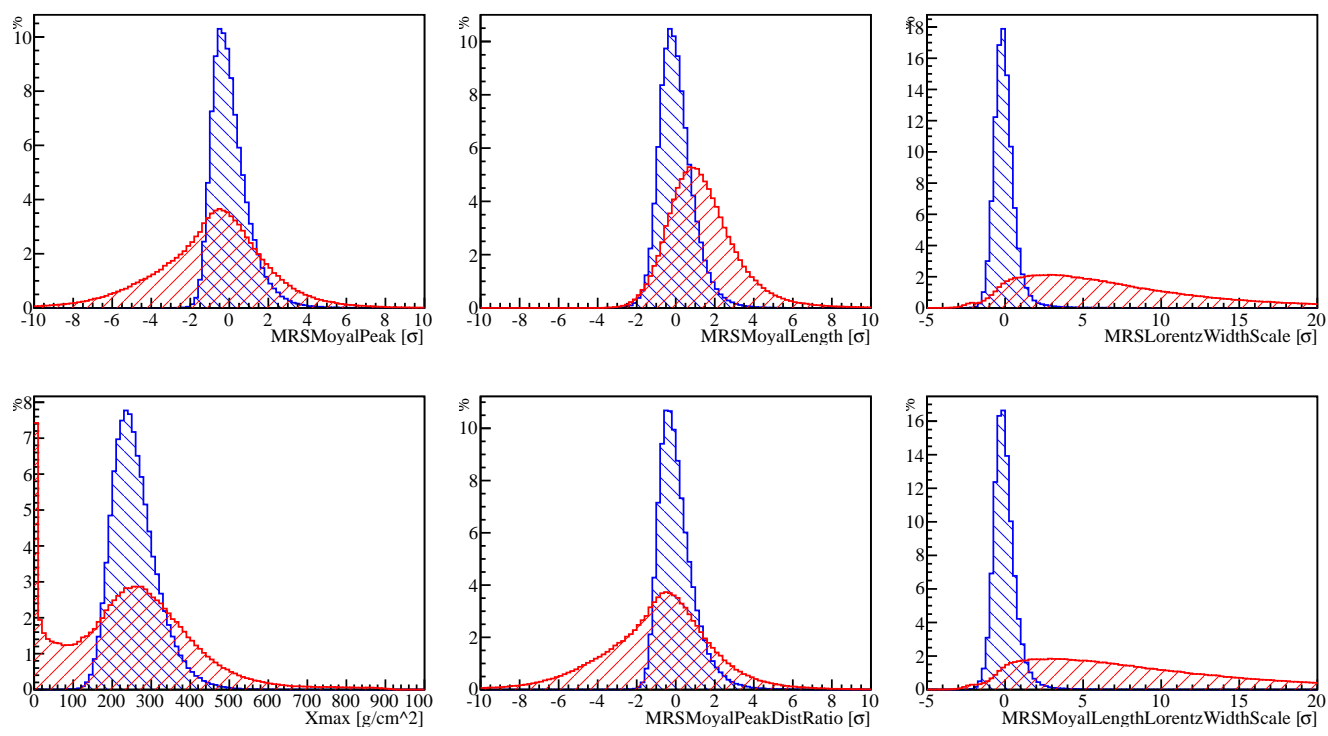

Figure 3: Distribution of new GRASP parameters for MC gamma (blue) and OFF data (red) with reconstructed energies between $(0.5-1.0) \mathrm{TeV}$ in the zenith angle range of $(15-25)^{\circ}$.

- Shower maximum Xmax, calculated from Moyal peak and reconstructed impact distance

- Ratio of Moyal peak $p$ to impact distance $d$

The parameter for the shower maximum Xmax is averaged over each telescope. Since the other parameters can be calculated for each participating telescope $i$ as well they will be mean scaled in the same manner as for the Hillas Parameters described in [8]. The scaled parameters are defined as $\mathrm{SCMoyalPeak}_{i}=\left(\right.$ MoyalPeak $_{i}-\left\langle\right.$ MoyalPeak $\left.\left._{i}\right\rangle\right) / \sigma_{i}$, with $\sigma_{i}$ being the spread of the expected Moyal Peak. The mean reduced scaled Moyal Peak (MRSMoyalPeak) can then be calculated as the average SCMoyalPeak over all telescopes:

$$
\text { MRSMoyalPeak }=\frac{1}{\sum_{i \in N_{\text {tel }}} \omega_{i}} \cdot \sum_{i \in N_{\text {tel }}}\left(\mathrm{SCMoyalPeak}_{i} \cdot \omega_{i}\right),
$$

The weighting factor $\omega_{i}$ takes into account the accuracy of the $\gamma$-ray simulations, defined as $\omega_{i}=$ $\left\langle\text { MoyalPeak }_{i}\right\rangle^{2} / \sigma_{i}^{2}$. In this way the multiplicity of the air shower event is naturally incorporated and the training of boosted decision trees can be handled more easily. It should be noted, however, that some of these parameters are highly correlated, depending on the energy band. The major advantage of boosted decision trees are that it treats non-linear correlations and is robust against weak variables. This justifies the above step of introducing new parameters that might not turn out to help in improving the $\gamma$-hadron separation. Following the same principle as described in [7] the training is done in seven zenith bands between $0^{\circ}$ and $55^{\circ}$, six energy bands between 0.1 and $100 \mathrm{TeV}$ and four offset bands of $\left\{0.5^{\circ}, 1^{\circ}, 1.5^{\circ}, 2^{\circ}\right\}$. In each of those training bands the sample of MC $\gamma$-ray and hadronic background data are separated in a test and training sample, where checks are performed on the test sample after the training procedure. The parameters for the training are kept the same as described in [7] with the exception of the number of splits per tree which was 
increased to 150 since the number of parameters increased as well. After the training procedure for each zenith, energy and offset band the importance of each variable can be calculated as the rate of occurrence of a splitting variable during the training procedure, weighted by the squared separation-gain and the number of events in the corresponding nodes [9]. In the energy range of $0.3 \mathrm{TeV}$ to $0.5 \mathrm{TeV}$ the Moyal Length times Lorentz width scaling factor is the most important variable at zenith angles larger than $40^{\circ}$, while Xmax is equally important at all zenith angles but especially at energies below $1 \mathrm{TeV}$.

\subsection{Comparison of the cut sets}

The optimisation of the $\gamma$-ray selection cuts are obtained by maximising the significance $\sigma$ (defined in [10]) depending on a few parameters. For the H.E.S.S. std $\zeta$ Hillas analysis [7], the ImPACT method [11] as well as for GRASP+ImPACT there are only two. The first is the $\theta^{2}$ and is defined as the squared angular distance between the assumed source position and the reconstructed shower direction. The second is the $\gamma$-efficiency $\varepsilon(\zeta)$ for every $\zeta$ value ( $\zeta$ is the output of the BDT classifier). This $\gamma$-efficiency is obtained from the independent test sample to predict the $\gamma$-efficiency of all possible $\zeta$ cuts in each zenith angle- and energy band. Since there are four different offset bands for GRASP+ImPACT the $\varepsilon(\zeta)$ is interpolated between the bands for an event with a given offset. The optimisation was done for a source with $10 \%$ of the integrated Crab flux above 200

\begin{tabular}{|c|c|c|c|}
\hline Configuration & $\begin{array}{c}\varepsilon_{\gamma}(\zeta) \\
\text { Max }\end{array}$ & $\begin{array}{c}\theta_{\text {cut }}^{2} \\
\text { Max } \\
\text { (degrees }^{2} \text { ) }\end{array}$ & $\begin{array}{c}\text { Size } \\
\text { Min } \\
\text { (p.e. })\end{array}$ \\
\hline std $\zeta$ Hillas & 0.84 & 0.0125 & 60 \\
std ImPACT & 0.83 & 0.005 & 60 \\
std GRASP+ImPACT & 0.85 & 0.0065 & 60 \\
\hline
\end{tabular}

Table 1: Selection cuts comparison optimised for the standard configuration.

$\mathrm{GeV}$ with a spectral index of $\Gamma=2.6$ (std cuts). Table 1 summarizes the results.

\section{Performance and Sensitivity}

Figure 4 compares the sensitivity (left) and differential sensitivity (right) for GRASP+ImPACT with ImPACT and the std $\zeta$ Hillas analysis. The left side illustrates the required observation time for a detection signal with more than $5 \sigma$ above background of a point source for a source like the Crab Nebula with a spectral index of $\Gamma=2.63$. For a source at $20^{\circ}$ zenith angle and $0.5^{\circ}$ offset with a flux of only $1 \%$ of the Crab flux $\left(3.45 \times 10^{-7}\right.$ photons $\left./ \mathrm{m}^{2} / \mathrm{s} / \mathrm{TeV}\right)$ the required observation time is reduced by a factor of 2.6 over the std $\zeta$ Hillas analysis and about $12 \%$ over the ImPACT method $(\Gamma=2.63)$. The right side shows that below some $300 \mathrm{GeV}$ the sensitivity is improved up to a factor of almost 2.3 and above $2 \mathrm{TeV}$ up to a factor of 1.2. It is calculated for an observation time of 25 hours for a detection signal with more than $5 \sigma$ above background of a point source for a source like the Crab Nebula with a spectral index of $\Gamma=2.63$. 

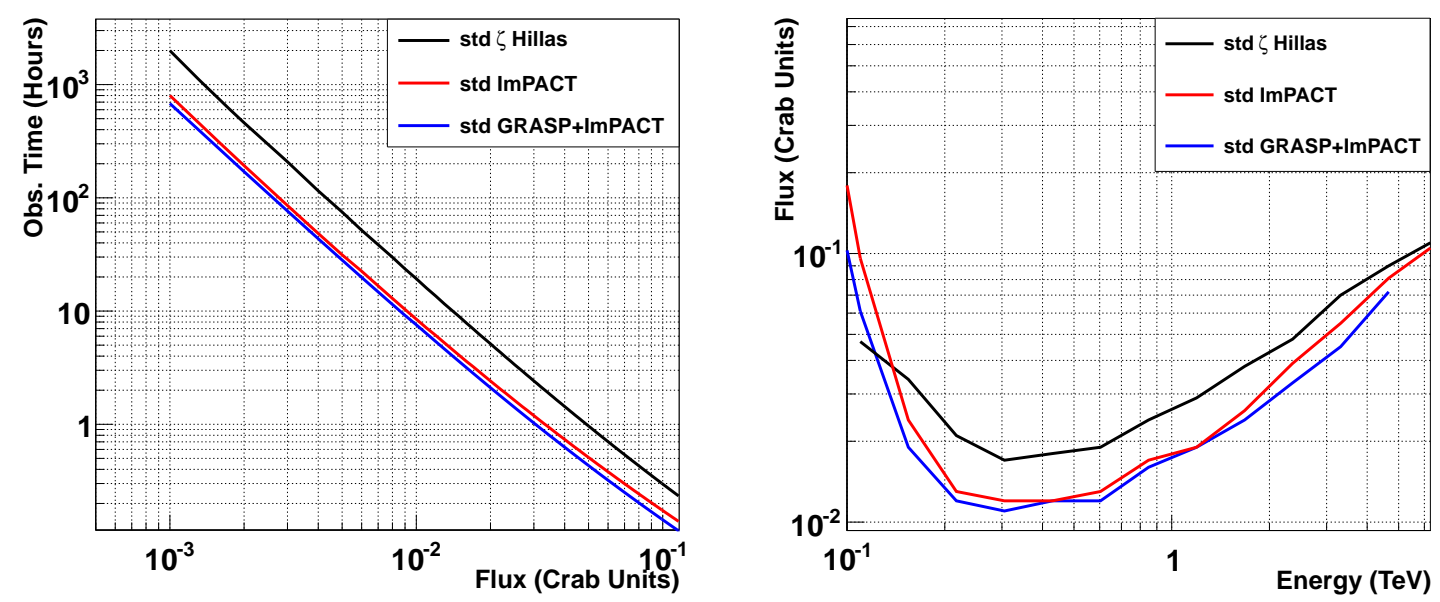

Figure 4: Sensitivity (left) and differential sensitivity (right) of H.E.S.S. Phase I for GRASP+ImPACT, ImPACT only and std $\zeta$ as a function of units of the Crab flux.

\section{Spectral source analysis}

The optimised standard cuts were applied to the blazar-type Active Galactic Nucleus (AGN) PKS 2155-304 data set of about 18 hours and a spectrum was extracted. Figure 5 compares the GRASP+ImPACT flux points with the points calculated by the ImPACT method alone. It shows an excellent agreement between both results. Table 2 summarizes the event statistics and significance for the different analysis methods.

\begin{tabular}{|c|c|c|c|}
\hline Configuration & $\mathrm{N}_{\text {on }}$ & $\alpha \mathrm{N}_{\text {off }}$ & $\sigma$ \\
\hline std $\zeta$ Hillas & 5177 & 564 & 105 \\
std ImPACT & 4764 & 219 & 128 \\
std GRASP+ImPACT & 7017 & 375 & 149 \\
\hline
\end{tabular}

Table 2: Comparison of the standard $\zeta$ Hillas analysis with the ImPACT and GRASP+ImPACT method for PKS 2155 - 304. Summarizes the event statistics and significance (defined in [10]) for about 18 hours of data.

\section{Conclusion}

In this work the GRASP method is introduced and the existing multivariate analysis technique developed in [7] is extended with new classifying variables. In combination with ImPACT [11] the sensitivity of the H.E.S.S. Phase I instrument especially at the lower energies, where the improved background rejection of GRASP is at its strongest is significantly increased. Performance tests have also shown a highly increased separation power for the GRASP+ImPACT analysis compared to the std $\zeta$ Hillas analysis especially for sources with a spectral index compatible or steeper with that measured for the spectrum of the Crab nebula. 


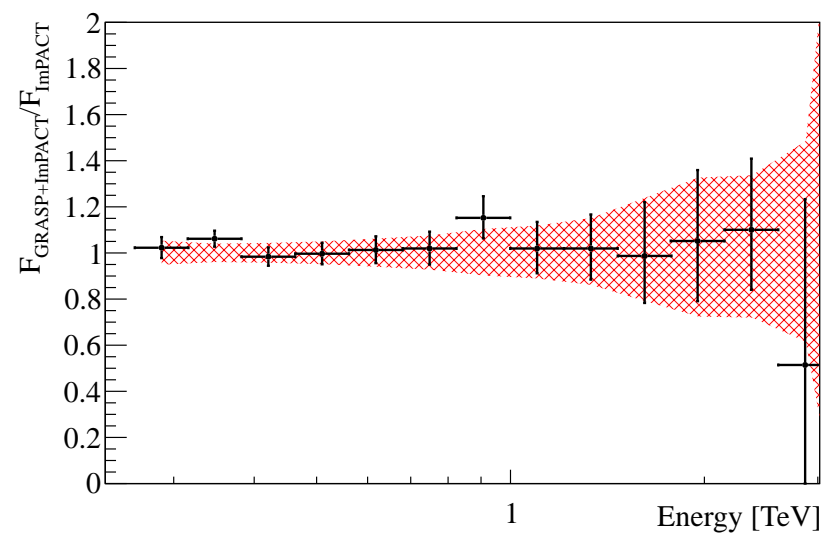

Figure 5: Ratio of flux points between GRASP+ImPACT and ImPACT analysis, which are obtained from 18 hours of H.E.S.S. PKS 2155 - 304 data. The error bars show the calculated errors for GRASP+ImPACT data, and the red shaded area for the ImPACT points.

\section{References}

[1] A. Hillas, In NASA. Goddard Space Flight Center 19th Intern. Cosmic Ray Conf., Volume 3

[2] D. Heck et al., Report FZKA 6019, Forschungszentrum Karlsruhe

[3] Hillert, A., PhD Thesis 2014, Ruprecht-Karls-Universität Heidelberg http://hdl.handle.net/11858/00-001M-0000-0023-E478-1

[4] Mathieu de Naurois and Loic Rolland, [arXiv:0907.2610]

[5] James, F. and Roos, M., Computer Physics Communications 1975, Volume 10, Issue 6

[6] Rene Brun and Fons Rademakers, New Computing Techniques in Physics Research V, ROOT - An object oriented data analysis framework

[7] S. Ohm et al., Astroparticle Physics 2009, (Volume 31)

[8] F.A. Aharonian et al., Astron. \& Astrophys. 457

[9] Breiman, L., et al., Classification and Regression Trees (Wadsworth, Stamford, 1984)

[10] Li, T.-P. and Ma, Y.-W., 1983, ApJ, 272, 317

[11] Parsons, R.D. and Hinton, J.A., Astroparticle Physics 2014, (Volume 56) 